\title{
Mimiviridae: clusters of orthologous genes, reconstruction of gene repertoire evolution and proposed expansion of the giant virus family
}

Natalya Yutin ${ }^{1}$, Philippe Colson ${ }^{2}$, Didier Raoult ${ }^{2}$ and Eugene $V$ Koonin ${ }^{1 *}$

\begin{abstract}
Background: The family Mimiviridae belongs to the large monophyletic group of Nucleo-Cytoplasmic Large DNA Viruses (NCLDV; proposed order Megavirales) and encompasses giant viruses infecting amoeba and probably other unicellular eukaryotes. The recent discovery of the Cafeteria roenbergensis virus (CroV), a distant relative of the prototype mimiviruses, led to a substantial expansion of the genetic variance within the family Mimiviridae. In the light of these findings, a reassessment of the relationships between the mimiviruses and other NCLDV and reconstruction of the evolution of giant virus genomes emerge as interesting and timely goals.

Results: Database searches for the protein sequences encoded in the genomes of several viruses originally classified as members of the family Phycodnaviridae, in particular Organic Lake phycodnaviruses and Phaeocystis globosa viruses (OLPG), revealed a greater number of highly similar homologs in members of the Mimiviridae than in phycodnaviruses. We constructed a collection of 898 Clusters of Orthologous Genes for the putative expanded family Mimiviridae (MimiCOGs) and used these clusters for a comprehensive phylogenetic analysis of the genes that are conserved in most of the NCLDV. The topologies of the phylogenetic trees for these conserved viral genes strongly support the monophyly of the OLPG and the mimiviruses. The same tree topology was obtained by analysis of the phyletic patterns of conserved viral genes. We further employed the mimiCOGs to obtain a maximum likelihood reconstruction of the history of genes losses and gains among the giant viruses. The results reveal massive gene gain in the mimivirus branch and modest gene gain in the OLPG branch.

Conclusions: These phylogenomic results reported here suggest a substantial expansion of the family Mimiviridae. The proposed expanded family encompasses a greater diversity of viruses including a group of viruses with much smaller genomes than those of the original members of the Mimiviridae. If the OLPG group is included in an expanded family Mimiviridae, it becomes the only family of giant viruses currently shown to host virophages. The mimiCOGs are expected to become a key resource for phylogenomics of giant viruses.
\end{abstract}

\section{Background}

The Nucleo-Cytoplasmic Large DNA Viruses (NCLDV) comprise a major, apparently monophyletic group of viruses that consists of 6 established virus families and a 7th putative family [1-3]. The NCLDV infect animals and diverse unicellular eukaryotes and either replicate exclusively within the so-called virus factories in the cytoplasm of the host cells $[4,5]$, or go through both

\footnotetext{
*Correspondence: koonin@ncbi.nlm.nih.gov

'National Center for Biotechnology Information, National Library of Medicine, National Institutes of Health, Bethesda, MD 20894, USA

Full list of author information is available at the end of the article
}

cytoplasmic and nuclear stages in their reproduction cycle [6].

With the exception of some viruses in the Phycodnaviridae family that do not encode their own RNA polymerase subunits and hence depend on the host for transcription, the NCLDV do not show strong dependence on the host replication or transcription systems for completing their replication [6,7]. This relative independence of the NCLDV from the host cells is consistent with the fact that these viruses encode many conserved proteins that mediate most of the processes essential for viral reproduction. These key proteins include DNA polymerases, primases, helicases, flap nucleases and

\section{Biomed Central}


DNA clamps that are responsible for DNA replication; Holliday junction resolvases and topoisomerases involved in genome DNA manipulation and processing; transcription factors that function in transcription initiation and elongation; ATPase pumps for DNA packaging; chaperones involved in the capsid assembly and the capsid proteins themselves $[1-3,8]$. Although only 5 genes are conserved in all NCLDV (with sequenced genomes), evolutionary reconstruction using maximum parsimony or maximum likelihood approaches mapped between 40 and 50 genes to the putative common ancestor of the NCLDV [2]. Given the compelling evidence in favor of the monophyly of the NCLDV, it has been recently proposed to formally recognize this group of viruses as a new taxon, the order Megavirales [9].

The best characterized family of the NCLDV is the Poxviridae that includes numerous viruses infecting animals including smallpox virus, the causative agent of one the most devastating human infectious diseases, and vaccinia virus, a classic model of molecular virology [10]. Recently, however, the group of the NCLDV that had attracted the most attention had been the family Mimiviridae that encompasses by far the largest known viruses [11-13]. The giant Mimivirus, the prototype of the family, was isolated from Acanthamoeba polyphaga and shown to possess $\sim 1.2 \mathrm{Mb}$ genome and encompass more than 1000 protein-coding genes [14]. Subsequently, 3 more genomes of related viruses have been sequenced, 2 of these even slightly larger than the Mimivirus genome [11,15-19]. In addition, approximately 20 mimiviruses have been detected through genomic and proteomic surveys but have not yet been characterized in detail [20]. Most of the currently identified mimiviruses infect the freshwater protist (and an opportunistic human pathogen) Acanthamoeba but the current genome size record holder, Megavirus chiliensis, was isolated from ocean water although its specific host remains unknown [21]. Recently a giant (albeit somewhat smaller than the previously isolated mimiviruses, with a $700 \mathrm{~Kb}$ genome) virus has been isolated from the marine flagellate Cafeteria roenbergensis (and accordingly designated CroV after Cafeteria roenbegensis virus) [22,23]. Phylogenetic analysis of the core NCLDV genes indicated that, among the other NCLDV, CroV was the closest relative of the mimiviruses and could be classified as a distant member of the family Mimiviridae [22,24]. Furthermore, numerous sequences homologous to mimivirus genes have been identified in marine metagenomic samples indicating that mimiviruses are common in these habitats [25,26]. Taken together, these findings indicate that Mimiviridae is an expansive family of giant viruses the true diversity of which remains largely untapped.

In addition to all the core NCLDV genes, members of the family Mimiviridae possess many genes the presence of which in viruses is unexpected, in particular genes encoding components of the translation systems such as aminoacyl-tRNA synthetases and translation factors $[14,21]$. The discovery of these genes that comprise parts of the core molecular machinery of all cellular life forms but are uncharacteristic of viruses fueled the debate on the controversial possibility that mimiviruses represent a "fourth domain of life" [9,14,24,27-29].

A notable feature of giant viruses is that they harbor their own mobilome, a collection of diverse selfish elements that depend on a giant virus for their reproduction. In addition to self-splicing introns and inteins, mimiviruses support the replication of transpovirons, a distinct type of linear plasmids, and virophages, small viruses that replicate within the intracellular factories of the host giant virus [30,31]. The first discovered virophage, dubbed Sputnik, is a parasite of the Mamavirus and closely related mimiviruses, and is an icosahedral virus with an approximately 20 kilobase dsDNA genome [16]. Subsequently, it has been shown that Sputnik can integrate into the genome of the host mimiviruses [30]. Two distinct virophages have been shown to infect CroV [32] and Organic Lake phycodnavirus [33]; these virophages resemble Sputnik in terms of the overall virion and genome structure but substantially differ in their gene repertoires.

As part of an effort to understand the evolutionary history and ultimately the origin of the giant viruses, we constructed Clusters of Mimivirus Orthologous Genes (mimiCOGs) and reassessed the relationship of the family Mimiviridae with the other NLCDV. The result is a potential major expansion of the family Mimiviridae that is shown to include several viruses previously classified as members of Phycodnaviridae.

\section{Results and discussion}

\section{Comparative genomics of the putative expanded family} Mimiviridae

In the course of phylogenomic study of the NCLDV, we noticed that in sequence database searches the proteins from some large DNA viruses assigned to the family Phycodnaviridae, namely Organic Lake phycodnaviruses [33] and Phaeocystis globosa viruses $12 \mathrm{~T}$ and 14 T [34,35] produce a substantially greater number of best hits into mimiviruses than into phycodnaviruses (Figure 1, Additional file 1)

To further investigate the evolutionary provenance of these poorly characterized giant viruses (hereinafter OLPG, after Organic Lake and Phaeocystis globosa viruses), we conducted an in depth phylogenomic analysis of the previously identified and putative new members of the family Mimiviridae. To this end, we constructed clusters of orthologous genes (COGs [36,37]) from the genomes of 4 mimiviruses (Acanthamoeba castellanii mamavirus, Acanthamoeba polyphaga mimivirus, Megavirus chiliensis, 


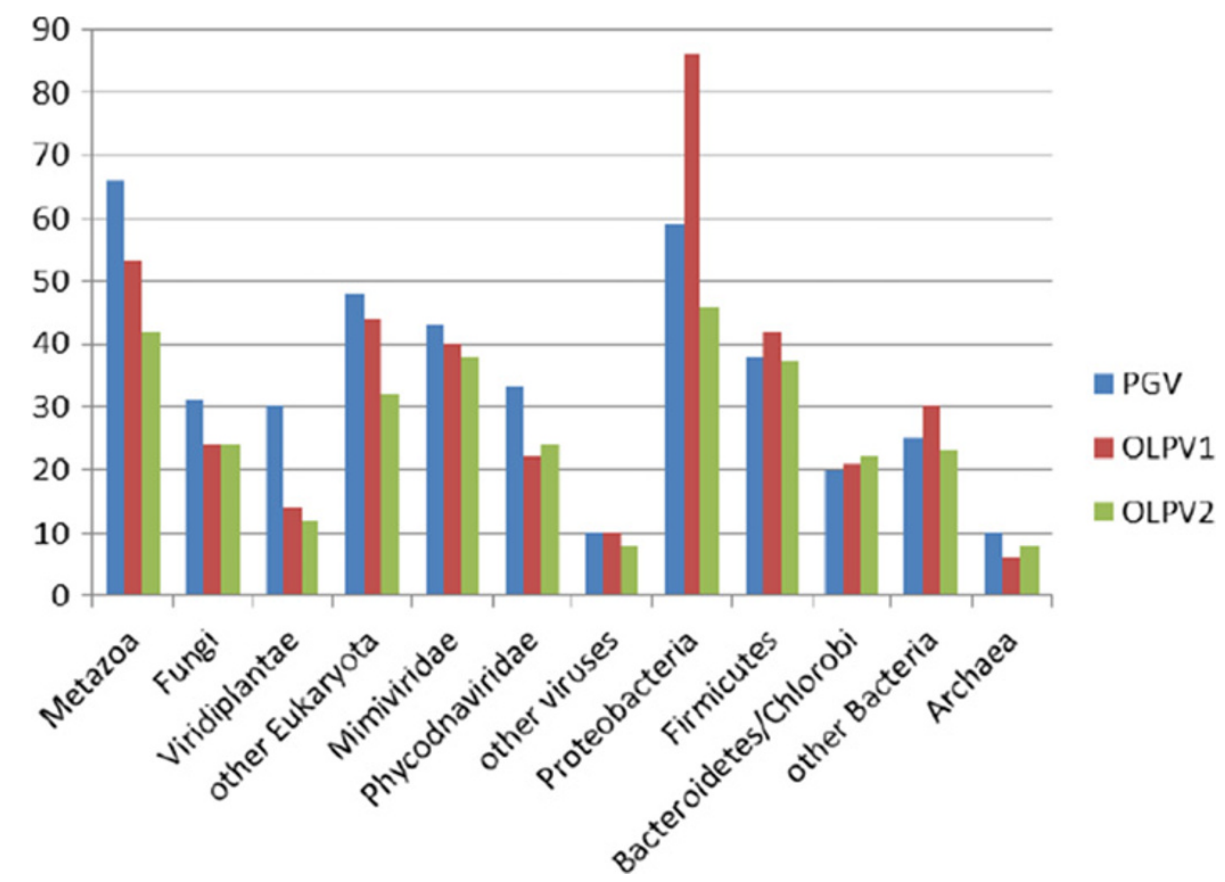

Figure 1 Phyletic distribution of Refseq best BLAST hits of $P$. globosa virus 12 T (PGV), Organic Lake phycodnavirus 1 (OLPV1), and Organic Lake phycodnavirus 2 (OLPV2). The family Mimiviridae included C. roenbergensis virus BV-PW1, A. polyphaga mimivirus, and Megavirus chiliensis. Phycodnaviridae include: Bathycoccus sp. RCC1 105 virus BpV1, Ectocarpus siliculosus virus 1, Emiliania huxleyi virus 86, Feldmannia species virus, Micromonas sp. RCC1109 virus MpV1, Ostreococcus lucimarinus virus OIV1, O. tauri virus 1, O. tauri virus 2, O. tauri virus OsV5, and Chloroviruses.

and Moumouvirus), CroV, and 3 OLPG [Organic Lake phycodnavirus 1, Organic Lake phycodnavirus 2 (these two genomes are still incomplete) and Phaeocystis globosa virus $12 \mathrm{~T})]$. The gene products encoded in these 8 genomes were retrieved from GenBank yielding a total of 5,677 protein sequences. These viral proteins were grouped into clusters of likely orthologs using a modified COG procedure [38] (see Methods for details). Clusters were manually edited and annotated using the results of RPS-BLAST and PSI-BLAST searches for the constituent proteins (Additional file 2 and see Methods). This procedure yielded 898 clusters of candidate orthologous genes from the putative expanded family Mimiviridae (hereinafter mimiCOGs). The mimiCOGs then were merged into the previously constructed clusters of orthologous genes for all NCLDV (NCVOGs [8]) (see Methods for details).

Fifty-two genes are present in all 8 genomes of the members of the putative expanded family Mimiviridae (Table 1). In addition, 10 other genes are missing in one or two OLPG genomes but present in all genomes of the Mimiviridae; these genes also might be conserved in all analyzed viruses given the incompleteness of the OLPG genomes. These conserved genes include mostly the core genes with essential functions in viral replication and virion morphogenesis that are also widely represented in other NCLDV and are likely to be ancestral to this entire group of viruses [8]. However, beyond the core gene set, the genes conserved in the Mimiviridae and the OLPG encode several additional proteins implicated in viral replication (e.g. RNAse $\mathrm{H}$, two paralogous small subunits of replication factor $\mathrm{C}$ and topoisomerase II) and transcription (e.g. TATA-binding protein) as well as proteins implicated in modification of host cell systems during virus infection such as a homolog of translation elongation factor $2 \mathrm{E}$ and ubiquitin $\mathrm{C}$-terminal hydrolase (Table 1). Apart from the 52 genes that are conserved in all analyzed viral genomes, the majority of the mimiCOGs are Mimivirus-specific and missing OLPG (Figure 2). The set of OLPG-specific genes is considerably smaller. Interestingly, these genes encode several functions that have not been previously identified in viruses including the first gene for proteorhodopsin identified in virus genomes [39].

A Neighbor-Joining gene content tree $[8,40]$ was constructed from gene presence-absence patterns in 1,723 mimiCOGs and NCVOGs (Figure 3). In this tree the OLPG forms a clade with the Mimiviridae including $\mathrm{CroV}$ as the outgroup to the mimiviruses sensu strictu. Thus, the similarity of the gene repertoires is compatible with the common ancestry of the OLPG and the Mimiviridae. A maximum likelihood reconstruction of the evolution of the NCLDV [8] assigned nearly 50 viral genes to the ancestral core that presumably dates back 
Table 1 Conserved proteins of the putative extended Mimiviridae family

\begin{tabular}{|c|c|c|c|}
\hline \multicolumn{4}{|c|}{ Proteins present in all 8 Mimiviridae genomes } \\
\hline CLS10031 & A $1 \mathrm{~L}$ transcription factor VLTF-2 & CLS10052 & Proliferating cell nuclear antigen \\
\hline CLS10071 & A2L transcription factor VLTF-3 & CLS10035 & protein disulfide Isomerase/thioredoxin family \\
\hline CLS10199 & asnB, asparagine synthetase B & CLS10216 & putative DNA-directed RNA polymerase II subunit N \\
\hline CLS10039 & capsid protein & CLS10047 & replication factor $C$ small subunit \\
\hline CLS10262 & D5-like helicase-primase & CLS10049 & replication factor C small subunit \\
\hline CLS10015 & DEAD/SNF2-like helicase or ATP-dependent RNA helicase & CLS10258 & ribonuclease $\mathrm{H}$ \\
\hline CLS10089 & DNA directed RNA polymerase subunit $L$ & CLS10041 & ribonuclease III \\
\hline CLS10259 & DNA mismatch repair ATPase MutS & CLS10130 & ribonucleosidediphosphatereductase large subunit \\
\hline CLS10104 & DNA polymerase elongation subunit family B & CLS10252 & ribonucleosidediphosphatereductase small subunit \\
\hline CLS10201 & DNA topoisomerase IB & CLS10028 & TATA-box-binding protein \\
\hline CLS10230 & DNA topoisomerase II & CLS10057 & Transcription factor S-\| (TFIIS)-domain-containing protein \\
\hline CLS10090 & DNA-dependent RNA polymerase subunit Rpb9/M & CLS10055 & transcription initiation factor IIB \\
\hline CLS10250 & DNA-directed RNA polymerase subunit 5 (RPB5) & CLS10011 & ubiquitin-conjugating enzyme E2 \\
\hline CLS10261 & DNA-directed RNA polymerase subunit 6 & CLS10214 & Ulp1-like protease \\
\hline CLS10076 & DNA-directed RNA polymerase subunit alpha & CLS10066 & W A18-like helicase \\
\hline CLS10053 & DNA-directed RNA polymerase subunit beta & CLS10068 & W A32 virion packaging ATPase \\
\hline CLS10249 & DNA-directed RNA polymerase subunit E' (RPB7) & CLS10218 & YqaJ-like viral recombinase \\
\hline CLS10024 & Erv1 / Alr family oxidoreductase & CLS10212 & hypothetical protein \\
\hline CLS10221 & eukaryotic translation initiation factor $4 \mathrm{E}$-like protein & CLS10222 & hypothetical protein \\
\hline CLS10086 & FtsJ-like methyltransferase & CLS10233 & hypothetical protein \\
\hline CLS10030 & Holliday junction resolvase & CLS10236 & hypothetical protein \\
\hline CLS10056 & metallopeptidase WLM & CLS10032 & hypothetical protein \\
\hline CLS10219 & mRNA capping enzyme & CLS10043 & hypothetical protein \\
\hline CLS10088 & NUDIX hydrolase & CLS10046 & hypothetical protein \\
\hline CLS10224 & poxvirus poly $(A)$ polymerase catalytic subunit-like protein & CLS10070 & hypothetical protein \\
\hline CLS10253 & probable ubiquitin carboxyl-terminal hydrolase & CLS10081 & hypothetical protein \\
\hline \multicolumn{4}{|c|}{ Genes missing in one or two OLPG genomes but present in all the other Mimiviridae genomes } \\
\hline CLS10059 & AAA family ATPase & CLS10009 & Lon domain protease \\
\hline CLS10021 & chaperone protein DnaJ & CLS10033 & patatin-like phospholipase \\
\hline CLS10022 & chaperone protein DnaJ & CLS10072 & Prolyl 4-hydroxylase \\
\hline CLS10082 & heat shock $70 \mathrm{kDa}$ protein & CLS10091 & thymidylate synthase \\
\hline CLS10042 & hypothetical protein & CLS10010 & XRN 5'-3' exonuclease \\
\hline
\end{tabular}

to the last common ancestor of all NCLDV although some of the ancestral genes were replaced with xenologs in the course of subsequent evolution [41].

\section{The Mimiviridae-OLPG clade in the phylogenetic trees of conserved NCLDV genes}

We used the mimiCOGs to conduct a new phylogenomic analysis of the ancestral NCLDV genes in an attempt to elucidate the evolutionary affinity of the OLPG (Additional file 3: Table S2). Phylogenetic trees were constructed for all clusters of orthologous genes that included the mimiviruses, OLPG and phycodnaviruses and for which the number of informative sites in the multiple sequence alignment was sufficient for phylogenetic analysis.

\section{Genes involved in DNA replication, recombination and repair}

Among 13 genes in this category, 7 are missing in both Phycodnaviruses and the OLPG, suggestive of parallel gene loss (See Additional file 3: Table S2). In the DNA polymerase B tree, the OLPG cluster with mimiviruses with 0.99 bootstrap support (Figure 4A). Three unclassified, partially sequenced viruses, Chrysochromulina ericina virus, Phaeocystis pouchetii virus, and Pyramimonas orientalis virus, also appear to belong to the OLPG group. 


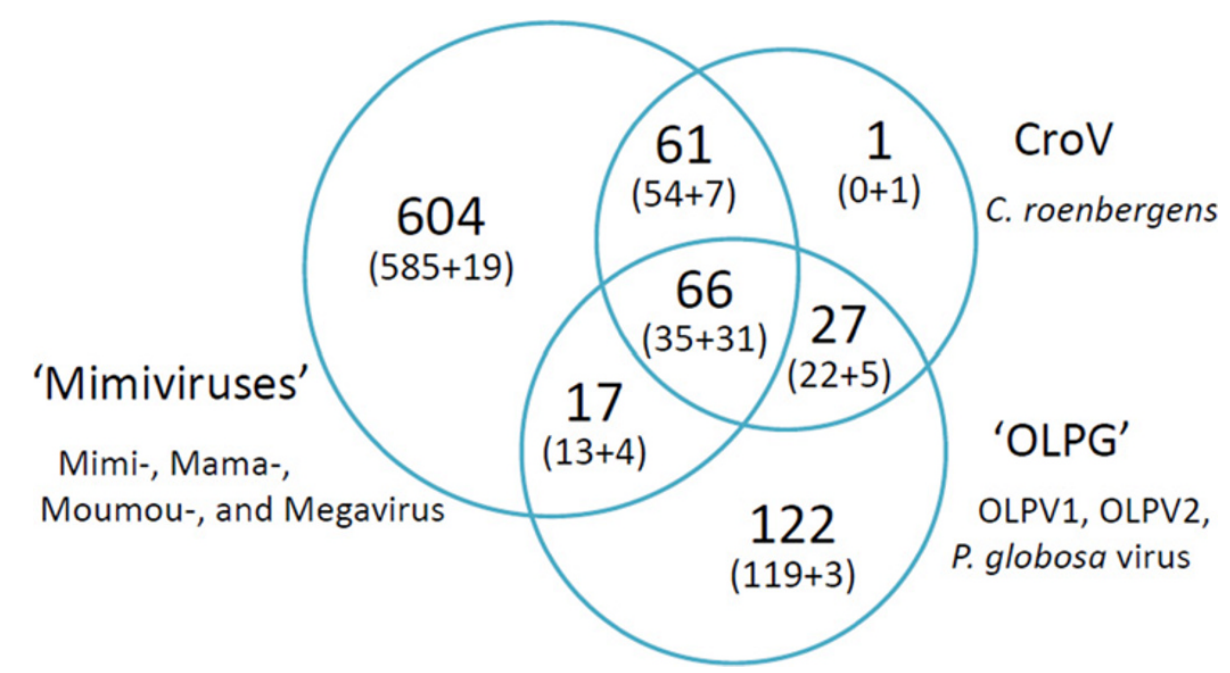

Figure 2 Virus species content of the mimiCOGs. The Venn diagram shows the numbers of mimiCOGs that are unique to and shared between three phyletic groups: Mimiviridae, CroV and OLPG.

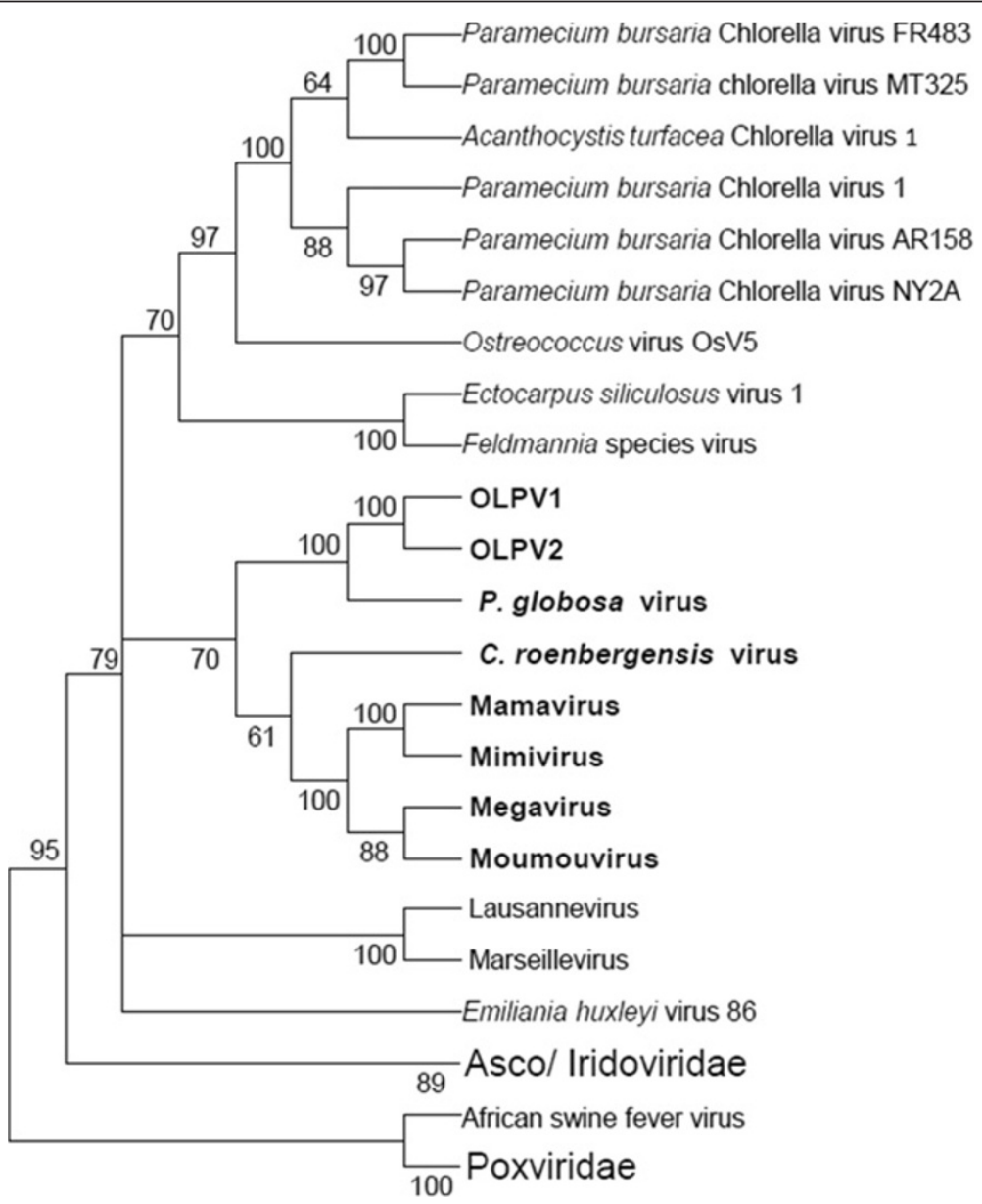

Figure 3 Neighbor-Joining gene content tree of the NCLDV. Bootstrap values were obtained by 1,000 resamplings of the initial patterns. 

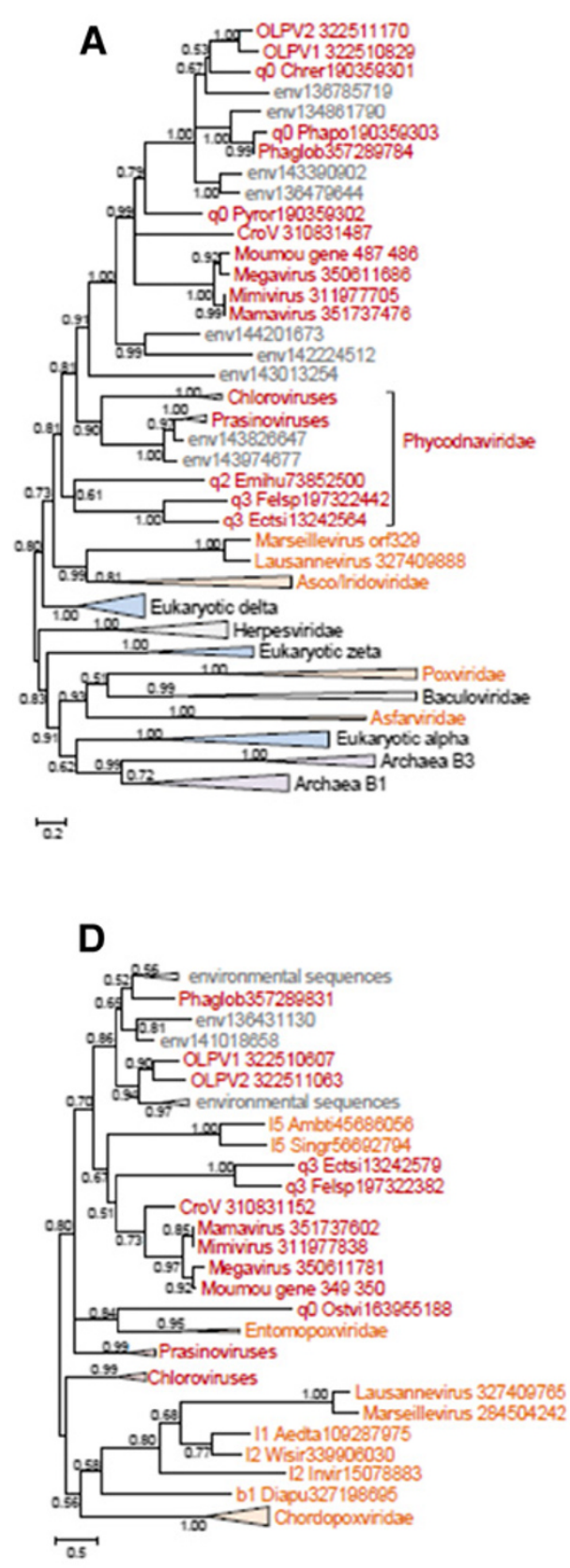

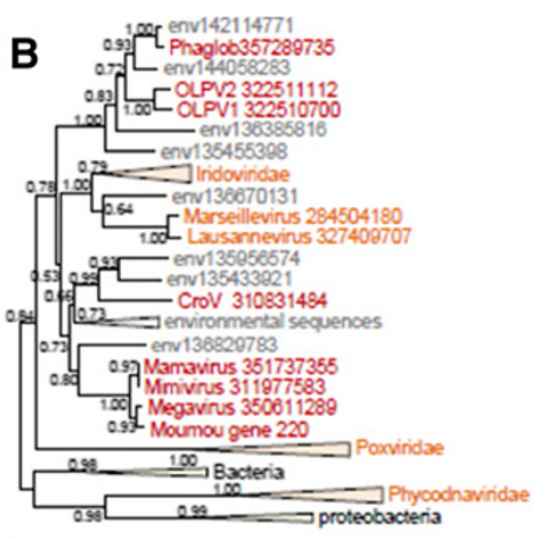

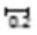
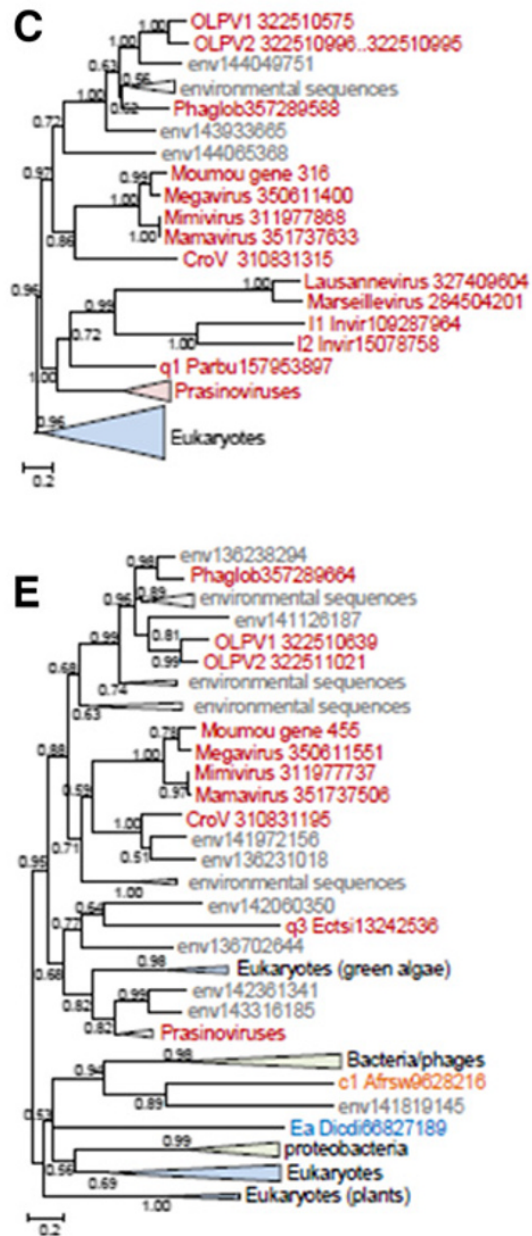

Figure 4 Maximum-Likelihood trees of ancestral NCLDV genes involved in DNA replication, recombination, and repair. A, DNA polymerase B, D5 primase-helicase. C, DNA topoisomerase II. D, Holliday junction (RuvC) resolvase. E, YqaJ-like recombinase. Branches with bootstrap support less than 0.5 were collapsed. For each sequence, the species name abbreviation and the gene identification numbers are indicated; env stands for "marine metagenome." Species abbreviations: CroV, C.roenbergensis virus; Moumou, Moumouvirus; OLPV1, Organic Lake phycodnavirus 1; OLPV2, Organic Lake phycodnavirus 2; Phaglob, P. globosa virus; Aedta, Invertebrate iridescent virus 3; Afrsw, African swine fever virus; Ambti, Ambystomatigrinum virus; Chrer, Chrysochromulina ericina virus; Diapu, Diadromuspulchellus ascovirus 4a; Dicdi, Dictyostelium discoideum AX4; 11_Invir, Invertebrate iridescent virus 3; 12_Invir, Invertebrate iridescent virus 6; Ostvi, Ostreococcus virus OsV5; Parbu, Paramecium bursaria Chlorella virus AR158; Phapo, Phaeocystis pouchetii virus; Pyror, Pyramimonas orientalis virus; Singr, Singapore grouper iridovirus; Wisir, Wiseana iridescent virus; Ectsi, Ectocarpus siliculosus virus 1; Emihu, Emiliania huxleyi virus 86; Felsp, Feldmannia sp virus. Taxa abbreviations: Ea, Amoebozoa; b1, Ascovirus; c1, Asfarviridae; 11, Chloriridovirus; 12, Iridovirus; 15, Ranavirus; q0, unclassified Phycodnaviridae; q1, Chlorovirus; q2, Coccolithovirus; q3, Phaeovirus. 

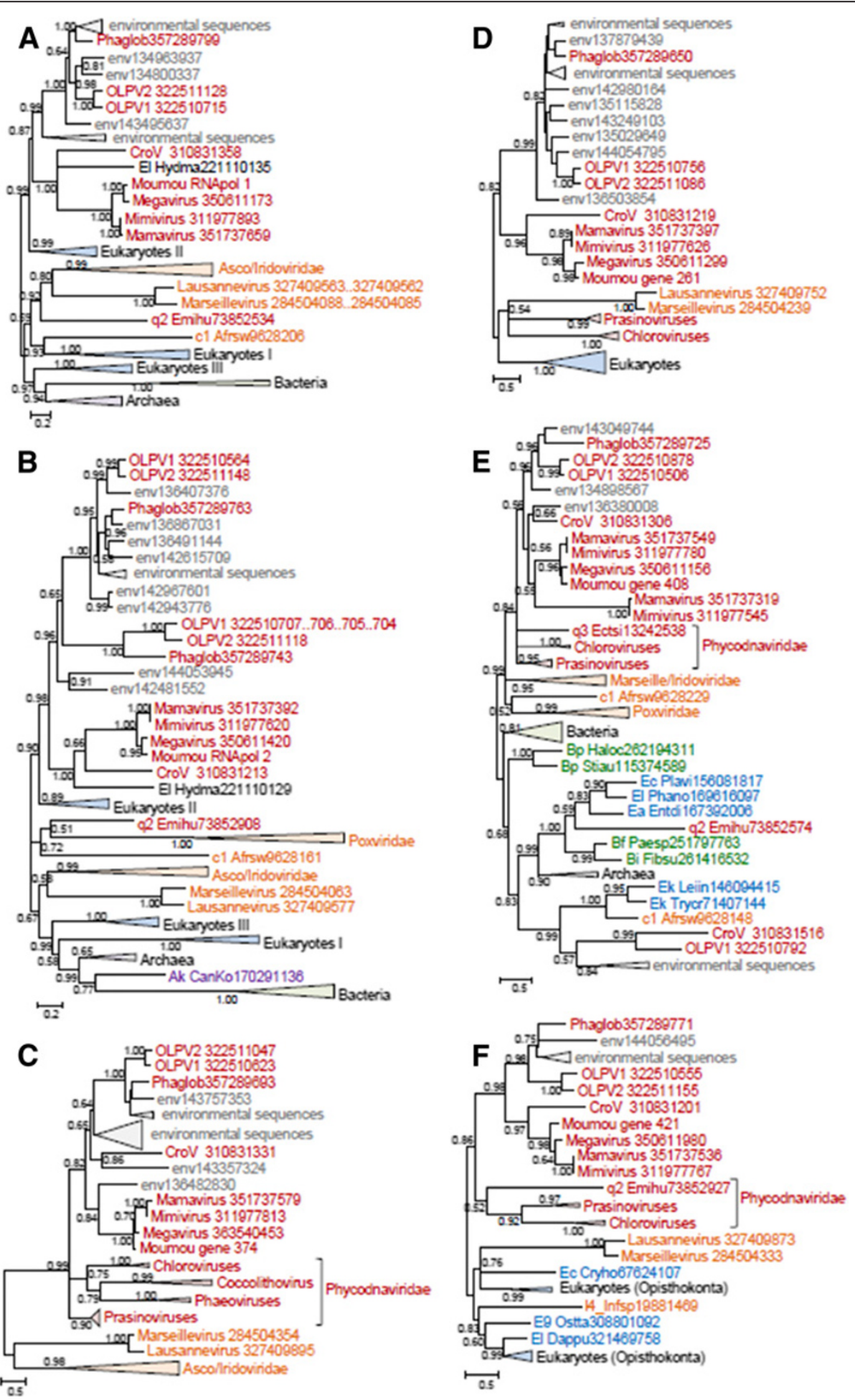

Figure 5 Maximum-Likelihood trees of ancestral NCLDV genes involved in transcription and RNA processing. A, RNA polymerase alpha subunit. B, RNA polymerase beta subunit. C, A2L transcription factor. D, Transcription initiation factor TFIIB. E, A18-like helicase. F, mRNA capping enzyme. Branches with bootstrap support less than 0.5 were collapsed. For each sequence, the species name abbreviation and the gene identification numbers are indicated; env stands for environmental sequences. Species abbreviations:CroV, C.roenbergensis virus; Moumou, Moumouvirus; OLPV1, Organic Lake phycodnavirus 1; OLPV2, Organic Lake phycodnavirus 2; Phaglob, P. globosa virus; Afrsw, African swine fever virus; CanKo, Candidatus Korarchaeum cryptofilum OPF8; Emihu, Emiliania huxleyi virus 86; Hydma, Hydra magnipapillata. Taxa abbreviations: Ak, Korarchaeota; El, Opisthokonta; c1, Asfarviridae; q2, Coccolithovirus. 
Phycodnaviruses in this tree are paraphyletic, and topology testing confidently rejects monophyly of OLPG with any one of the two branches of Phycodnaviruses. In contrast, the monophyly of phycodnaviruses is supported, with the respective tree having a slightly greater likelihood than the unconstrained DNAP tree; however, joining OLPG with the single Phycodnavirus branch is rejected as well (Additional file 4: Figure S3).

In the D5 helicase tree (Figure 4B), OLPG and mimiviruses are paraphyletic but form a well-supported clade with iridoviruses and Marseilleviruses whereas phycodnaviruses group with bacteria and bacteriophages, probably as a result of xenologous gene displacement [41].

The phylogenetic tree of DNA topoisomerase II contains a strongly supported OLPG-Mimiviridae clade (Figure 4C); the topology of this tree is nearly identical to that of the DNA polymerase tree. The tree of the YqaJ-like recombinase also supports the OLPG-Mimiviridae clade (Figure 4D). By contrast, in the tree of RuvC-like Holliday junction resolvases, the OLPG fail to cluster with either phycodnaviruses or mimiviruses (Figure 4E).

\section{Genes involved in transcription and RNA processing}

The RNA polymerase (RNAP) subunits alpha and beta have been lost in Phycodnaviruses. However, given that the NCLDV are polyphyletic in the phylogenies of both these genes [41], we constructed trees and examined the provenance of the OLPG. In both trees (Figure 5AB), OLPG and the Mimiviridae are monophyletic and group with Eukaryotic RNAP II. Notably, the RNAP beta gene is duplicated in OLPG. The phylogenies of other genes encoding proteins involved in transcription and RNA processing including the transcription factors A2_L and TFIIB, A18-like helicase, and capping enzyme (guanylyltransferase domain only because the methyltransferase domain is missing in phycodnaviruses) also showed monophyly of OLPG and the Mimiviridae (Figure 5C-F).

Among the genes encoding enzymes of nucleotide metabolism, only those for the two subunits of ribonucleotide reductase were amenable to phylogenetic analysis. The tree for the small subunit supports monophyly of OLPG-Mimiviridae (Figure 6A) whereas in the tree

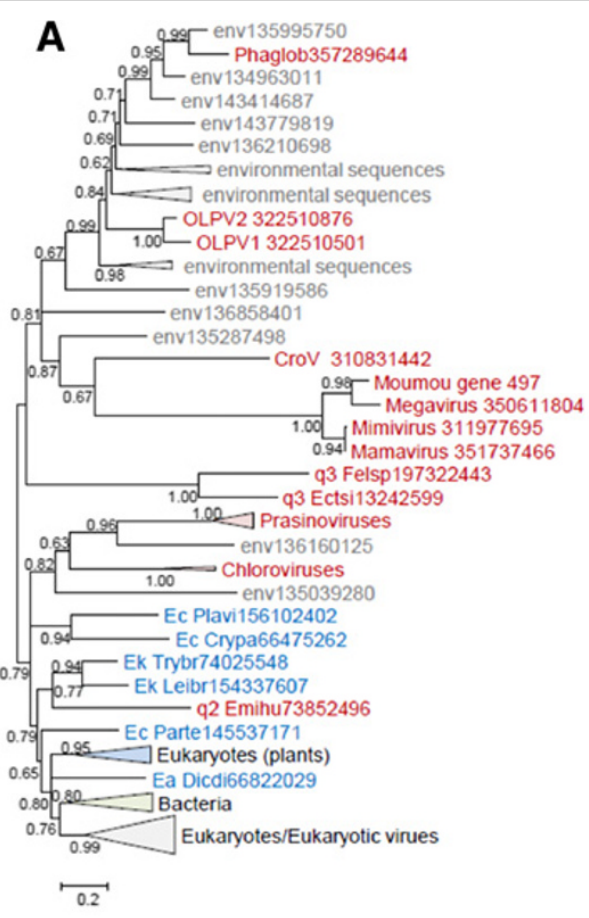

\section{B}



Figure 6 Maximum-Likelihood trees of ancestral NCLDV genes involved in nucleotide metabolism. A, Ribonucleoside diphosphate reductase small subunit. B, Ribonucleoside diphosphate reductase large subunit. Branches with bootstrap support less than 0.5 were collapsed. For each sequence, the species name abbreviation and the gene identification numbers are indicated; env stands for environmental sequences. Species abbreviations:CroV, C. roenbergensis virus; Moumou, Moumouvirus; OLPV1, Organic Lake phycodnavirus 1; OLPV2, Organic Lake phycodnavirus 2; Phaglob, P. globosa virus; Crypa, Cryptosporidium parvum lowa Il; Cyphe, Cyprinid herpesvirus 3; Dicdi, Dictyostelium discoideum AX4; Ectsi, Ectocarpus siliculosus 1; Emihu, Emiliania huxleyi virus 86; Felsp, Feldmannia sp virus; Leibr, Leishmania braziliensis; Musdo, Musca domestica salivary gland hypertrophy virus; Parte, Paramecium tetraurelia strain d4-2; Phatr, Phaeodactylum tricornutum CCAP 1055/1; Plavi, Plasmodium vivax Sal-1; Thaps, Thalassiosira pseudonana CCMP1335; Trybr, Trypanosoma brucei; Vibha, Vibrio harveyi 1DA3. Taxa abbreviations: Bp, Proteobacteria; E8, Stramenopiles; Ea, Amoebozoa; Ec, Alveolata; Ek, Kinetoplastida; k1, Herpesvirales; q2, Coccolithovirus; q3, Phaeovirus; zh, unclassified dsDNA viruses. 
for the large subunit the OLPG, the mimivirus and phycodnavirus branches are unresolved (Figure 6B).

The only phylogenetic tree that was obtained for a gene encoding a protein involved in virion morphogenesis, the A32-like DNA packaging ATPase, also supports the OLPG-Mimiviridae monophyly (Figure 7A).

The phylogenetic analysis of the Major Coat Protein (MCP) gene required a modified approach because the mimiviruses [18,19] as well as OLPG [33] encompass multiple paralogous MCP genes some of which are extremely diverged in sequence $[18,19]$, hampering the construction of robust phylogenetic trees. Therefore we first aligned all detected MCP sequences from Mimiviridae, OLPG, Phycodnaviridae, Iridoviridae and Marseilleviridae (the sequences from Asfarviridae and Poxviridae being in this case too distant) and constructed a preliminary phylogenetic tree. This tree was used to identify the fastest evolving MCP homologs (the longest branches) which were then removed from the sequence alignment that was when used to construct the final phylogenetic tree. In this MCP phylogeny, the OLPG-Mimiviridae clade was recovered with moderate statistical support (Figure 7B).

In addition, we examined the set of genes that are projected to the last common ancestor of the major branch of the NCLDV that consists of Iridoviridae, Marseilleviridae, Mimiviridae and Phycodnaviridae [8] and obtained phylogenetic trees for two of these genes, those for the Proliferating Cell Nuclear Antigen (PCNA)-like replication factor and ribonuclease III. Both trees support the OLPG-Mimiviridae monophyly (Figure 8AB).

\section{Reconstruction of the evolution of giant viruses}

The phyletic patterns of the amended NCVOGs were superimposed on the Neighbor-Joining gene content tree (Figure 3) and employed to produce a new maximum likelihood reconstruction of gene gain and loss in the NCLDV [8]. The reconstruction of the ancestral gene repertoires based solely on phyletic patterns is to be viewed with caution given the complexity of the evolution of the NCLDV that on some occasions apparently
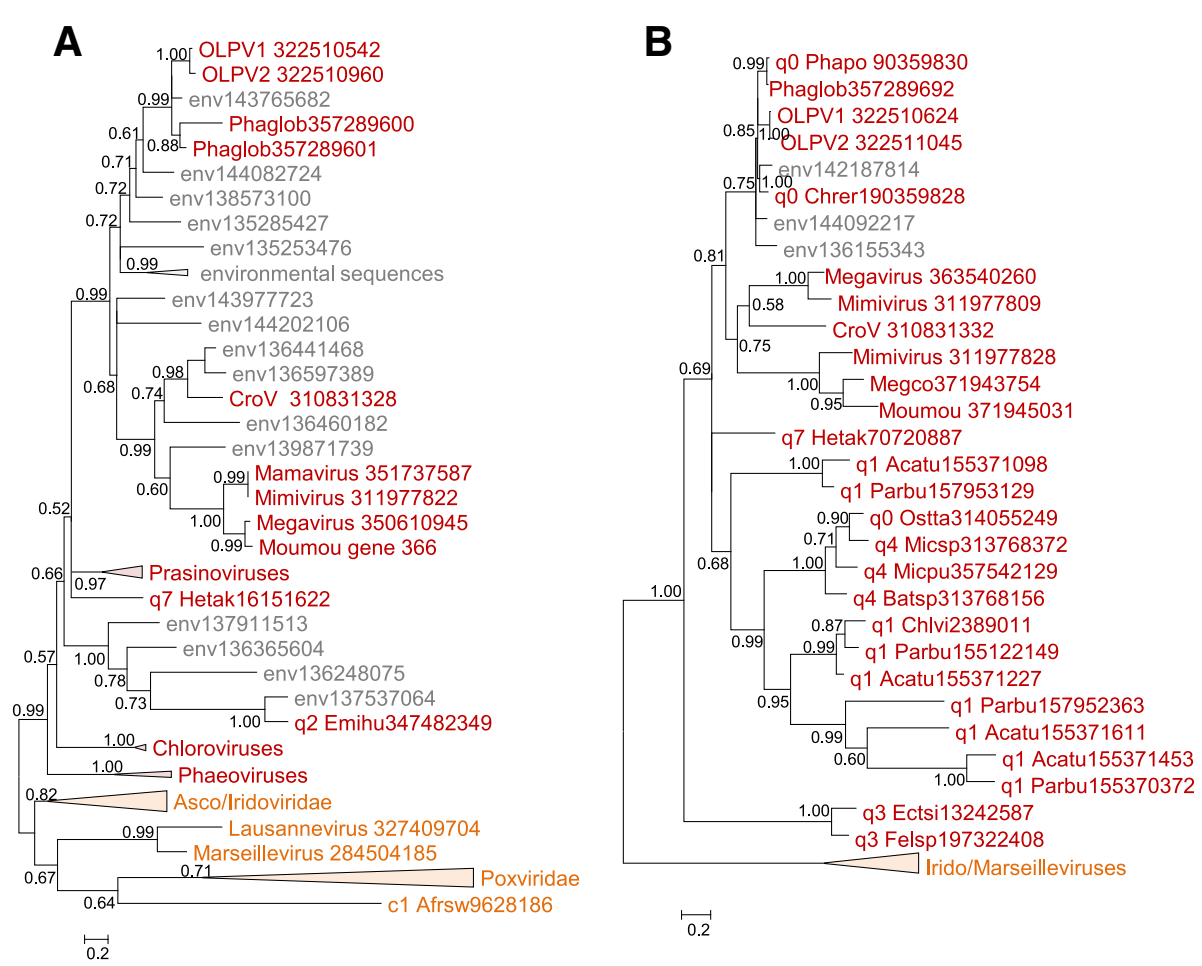

Figure 7 Maximum-Likelihood trees of two genes involved in virion structure and morphogenesis. A, A32 virion packaging ATPase. B, Major capsid protein. Branches with bootstrap support less than 0.5 were collapsed. For each sequence, the species name abbreviation and the gene identification numbers are indicated; env stands for "marine metagenome." Species abbreviations: Acatu, Acanthocystis turfacea Chlorella virus 1; Afrsw, African swine fever virus; Batsp, Bathycoccus sp. RCC1105 virus BpV1; Chlvi, Chlorella virus; Chrer, Chrysochromulina ericina virus; Crov , C. roenbergensis virus; Ectsi, Ectocarpus siliculosus virus 1; Emihu, Emiliania huxleyi virus 86; Felsp, Feldmannia species virus; Hetak, Heterosigma akashiwo virus 01; Megco, Megavirus courdo7; Micpu, Micromonas pusilla virus SP1; Micsp, Micromonas sp. RCC1109 virus MpV1; Moumou, Moumouvirus; OLPV1, Organic Lake phycodnavirus 1; OLPV2, Organic Lake phycodnavirus 2; Ostta, Ostreococcus tauri virus 2; Parbu, Paramecium bursaria Chlorella virus AR158; Phaglob, P. globosa virus; Phapo, Phaeocystis pouchetii virus. Taxa abbreviations: c1, Asfarviridae; q0, unclassified Phycodnaviridae; q1, Chlorovirus; q2, Coccolithovirus; q3, Phaeovirus; q4, Prasinovirus; q7, Raphidovirus. 

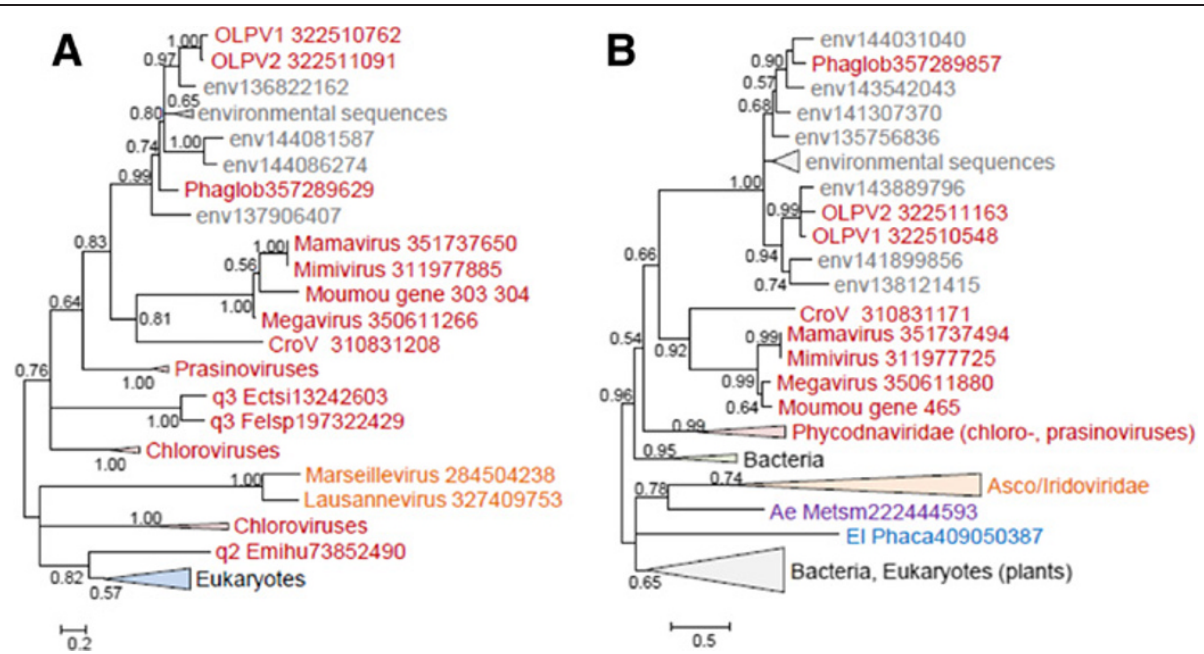

Figure 8 Maximum-Likelihood phylogenetic trees of two genes ancestral to the Mimi-,Asco/Irido-Phycodna-, and Marseilleviruses. A, Proliferating cell nuclear antigen. B, ribonuclease III. Branches with bootstrap support less than 0.5 were collapsed. For each sequence, the species name abbreviation and the gene identification numbers are indicated; env stands for "marine metagenome." Species abbreviations:CroV, C. roenbergensis virus; Moumou, Moumouvirus; OLPV1, Organic Lake phycodnavirus 1; OLPV2, Organic Lake phycodnavirus 2; Phaglob, P. globosa virus; Ectsi, Ectocarpus siliculosus virus 1; Emihu, Emiliania huxleyi virus 86; Felsp, Feldmannia species virus; Metsm , Methanobrevibacter smithii DSM 2375; Phaca, Phanerochaete carnosa. Taxa abbreviations: Ae, Euryarchaeota; El, Opisthokonta; q2, Coccolithovirus; q3, Phaeovirus.

involved parallel gains of homologous genes [41] as well as the inherent probabilistic nature of the reconstruction $[8,42]$. Nevertheless, the results clearly indicate some limited gene gain in the OLPG contrasted by massive gene gain in both the Mimiviridae branch and the mimiviruses sensu strictu, after their radiation from the common ancestor with the CroV (Figure 9AB; Additional file 4: Figure S4). This extensive gene gain in the mimiviruses, along with the considerable diversity of the gene repertoires even among closely related mimiviruses (Mimivirus,
Moumouvirus and Megavirus [30] implies a large, "open" pangenome of these giant viruses [43]. Conceivably, this expansive pangenome evolved through numerous acquisitions and exchanges of genes between diverse members of the vast intracellular microbiomes of phagotrophic amoeba that include bacteria, fungi and viruses $[30,44,45]$. Apparently, the extensive horizontal gene transfer within this microbiome results in mosaic gene repertoires of amoebal viruses as observed both in mimiviruses [14] and Marseillevirus [46]. Gene transfers
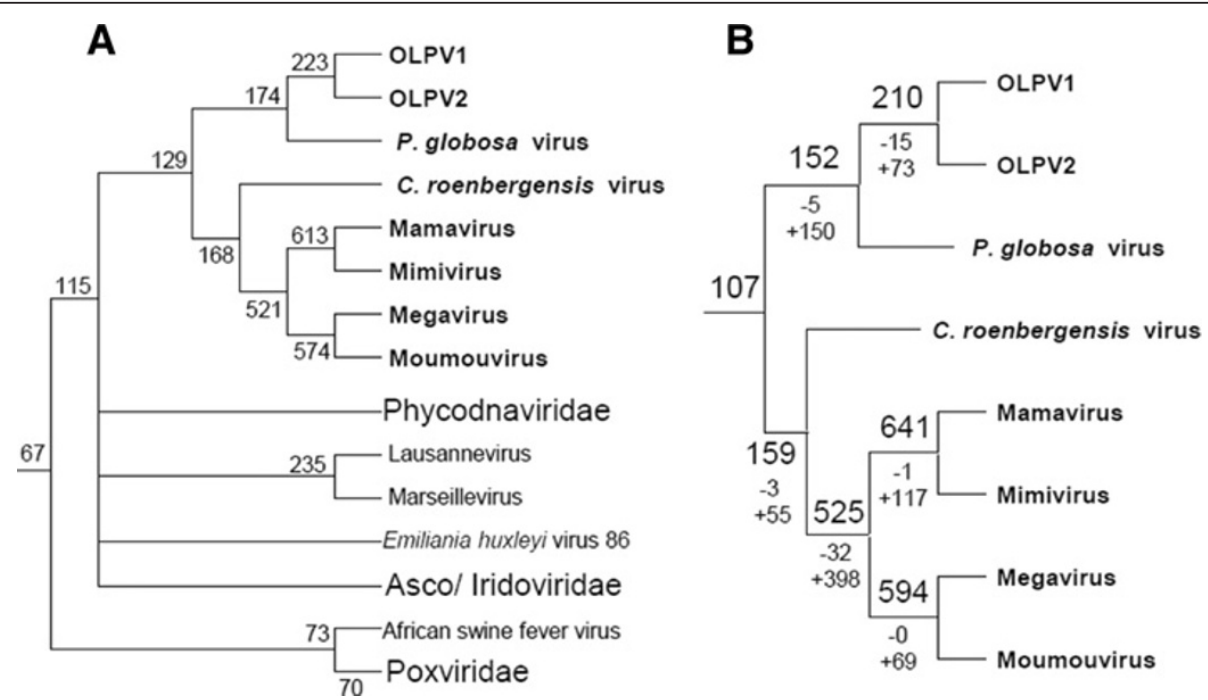

Figure 9 Maximum-Likelihood reconstruction of gene loss and gene gain events in the evolution of the NCLDV. The tree from Figure 3 was used as a guide for the reconstruction. A. The inferred numbers of genes present in each node are shown. B. Numbers of mimiCOGs present with the likelihood greater than 0.9. Numbers after plus and minus signs represent numbers of genes gained or lost since the previous node. 
are likely to be facilitated by the mobilome of the giant viruses that includes virophages as well as transpovirons, a distinct group of linear plasmids [30]. The OLPV, CroV and possibly other members of the extended family Mimiviridae that reproduce in hosts colonized by fewer microbes appear to possess smaller (pan)genomes and lower degrees of genomic mosaicism [22,33]. Thus, the size and diversity of the pangenomes of large viruses seem to strongly depend on the life styles of their hosts.

\section{Conclusions}

Taken together, the phylogenomic results presented here indicate that the OLPG are the sister group of the family Mimiviridae within the NCLDV phylogeny. This conclusion is supported by the topologies of the phylogenetic trees for most of the core NCLDV genes that show monophyly of OLPG and the mimiviruses (Figures 4, 5, 6, 7, 8 and Additional file 3: Table S2). Although some of the phylogenies are poorly resolved, none of them shows clustering of the OLPG with or within the phycodnaviruses. Moreover, for some of the core NCLDV genes, conservative statistical tests reject affiliation of OLPG with Phycodnaviruses. Given that the OLPG, at least so far, are a group with limited diversity, it seems plausible that eventually the family Mimiviridae is expanded to include these viruses. Alternatively, OLPG could become a new family within the proposed order Megavirales [9].

The OLPG encompass few genes encoding translation system components that are one of the signatures of the mimivirus genomes [14,21] (the only translation-related gene that was apparently acquired by the common ancestor of the OLPG and the mimiviruses is the homo$\log$ of the elongation factor $2 \mathrm{E}$ ) indicating that these genes largely were acquired by an ancestral mimivirus.

An Organic Lake "phycodnavirus" has been identified as a host to a distinct virophage (OLV) [33] that is distantly related to the Sputnik virophage infecting mimiviruses $[16,31]$ and the Mavirus virophage infecting $\mathrm{CroV}$ [32]. The findings described here indicate that so far only viruses within the (extended) family Mimiviridae support the reproduction of virophages. Recently, numerous sequences of putative virophages have been assembled from metagenomics sequences originating from diverse environments [47]. In particular, 4 complete virophage genomes distantly related to the OLV have been assembled from Yellowstone Lake metagenomic data. The presents results lead us to hypothesize that these novel virophages also infect member of the family Mimiviridae, in particular still unknown representatives of the OLPG group.

Finally, it is worth noting that the mimiCOGs developed in the course of this work are expected to become a key resource for a comprehensive phylogenomic study of the giant viruses, and in particular a full assessment of the fourth domain hypothesis.

\section{Methods}

\section{MimiCOG construction}

For the construction of mimiCOGs, the following genomes were downloaded from GenBank (http://www.ncbi. nlm.nih.gov/): Acanthamoeba polyphaga mimivirus (GI:311977355), Acanthamoeba castellanii mamavirus (GI:351737110), Megavirus chiliensis (GI:350610932), Cafeteria roenbergensis virus BV-PW1 (GI:310830989), Phaeocystis globosa virus 12 T (GI: 357289534), Organic Lake phycodnavirus 1 (GI:322510471),Organic Lake phycodnavirus 2 (GI:322510873), Marseillevirus (GI:284504040), and Lausannevirus (GI:327409548). The complete dataset consisted of 6,548 protein sequences. The mimiCOGs were constructed as previously described [38]. Briefly, the procedure included the following steps: 1) Initial clusters based on triangles of symmetrical best hits were constructed using a modified COG algorithm using as the input the results of all-against-all BLASTP [48] comparison; 2) Multiple alignments of the initial cluster members were constructed using the MUSCLE program [49]. The alignments were used to generate position-specific scoring matrices (PSSM) for a PSIBLAST search [48] against the original protein dataset. Significantly similar proteins were added to the corresponding clusters; 3 ) Clusters with nearly complementary phyletic patterns and high inter-cluster sequence similarity were manually examined and merged whenever appropriate; 4) The mimiCOGs were manually edited and annotated using annotations of Moumouvirus and Mamavirus proteins present and RPS-BLAST [50] and PSI-BLAST of other cluster members; 5) MimiCOGNCVOG correspondence was established by PSI-BLAST search initiated with PSSMs constructed from NCVOG alignments [8] against proteins included in the mimiCOGs. The mimiCOGs are available at ftp://ftp.ncbi.nih.gov/pub/ koonin/mimivirus/mimiCOGs.

\section{Neighbor-Joining tree based on the phyletic patterns}

Presence-absence matrices of mimiCOGs and corresponding NCVOGs were combined, whenever correspondence was established, and binarized yielding 584 patterns (see Additional file 5). Nineteen NCVOG patterns were amended by adding OLPG proteins that have not been included in the mimiCOGs based on the result of PSIBLAST searches initiated by NCVOG PSSMs against proteins used for mimiCOG construction. The remaining 727 NCVOGs and 393 mimiCOGs were considered nonoverlapping and added to the pool resulting in the total of 1,723 patterns. For each pair of species the number of clusters where each of them were present (N1 and $N 2)$ as well as the number of clusters where both species were 
present $(N U)$ were computed. The gene content similarity measure $(s)$ was calculated as $s=N \mathrm{U} / \operatorname{sqrt}(N 1 \times N 2)$ and converted to a distance measure $(d)$ as $d=-\ln (s)$ [8]. A neighbor-joining tree was constructed from the distance matrices using the NEIGHBOR program of Phylip 3.66 [51]. Bootstrap values were obtained by 1,000 resamplings of the 1,723 patterns.

\section{Multiple alignment and phylogenetic tree construction}

The sequences for phylogenetic analysis were collected using (i) BLAST searches against $\mathrm{nr}$ and environmental (env_nr) databases initiated by distant mimiCOG members; (ii) the corresponding NCVOG sequences [8]; and (iii) reference sequences used for the core NCVOG study [41]. Nearly identical sequences were eliminated using BLASTCLUST (http://www.ncbi.nlm.nih.gov/IEB/ ToolBox/C_DOC/lxr/source/doc/blast/blastclust.html). The sequences were aligned using MUSCLE [49]. All alignments were manually checked for the conservation of domain architecture and presence of diagnostic motifs. Positions including gaps in more than one-third of the sequences and positions with low information content were removed prior to tree computation [52]. A preliminary maximum-likelihood tree was constructed using the FastTree program with default parameters (JTT evolutionary model, discrete gamma model with 20 rate categories; [53]). The preliminary tree and the alignment were then used to determine the best substitution matrix using Prottest [54]. Final maximum-likelihood trees were constructed using TreeFinder (1,000 replicates, Search Depth 2 [55]), with the substitution matrix found to be the best for a given alignment. The Expected-Likelihood Weights (ELW) of 1,000 local rearrangements were used as confidence values of TreeFinder tree branches. For topology testing, whenever applicable, alternative (constrained) topologies were constructed and compared to the initial trees using TreeFinder. Approximately unbiased (AU) test $\mathrm{P}$ value cutoff 0.05 was used for rejecting tree topologies [56].

\section{Reconstruction of gene losses and gains}

The Neighbor-Joining gene content tree of the NCLDV and the gene presence-absence matrix for the mimiCOGs and NCVOGs were used to reconstruct the gene loss and gain events in the evolution of the NCLDV using the COUNT program [42], as previously described [8].

\section{Additional files}

Additional file 1: Annotation of the OLPG genes.

Additional file 2: The mimiCOGs.

Additional file 3: Table S2. Phyletic patterns and major inferred evolutionary events for ancestral NCVOGs.
Additional file 4: Topology testing results for selected phylogenetic trees of NCLDV genes.

Additional file 5: Phyletic patterns used for the reconstruction of gene gain and gene loss in the NCLDV.

Competing interests

The authors declare that they have no competing interests.

\section{Authors' contributions}

NY collected the data; NY, PC, DR and EVK analyzed the data; NY and EVK wrote the manuscript that was read and approved by all authors.

\section{Acknowledgments}

NY and EVK are supported by intramural funds of the US Department of Health and Human services (to the National Library of Medicine).

\section{Author details}

${ }^{1}$ National Center for Biotechnology Information, National Library of Medicine, National Institutes of Health, Bethesda, MD 20894, USA. ${ }^{2}$ URMITE, UM 63 CNRS 7278 IRD 198 INSERM U1095, Faculté de Médecine, Aix-Marseille University, 27 Boulevard Jean Moulin, Marseille Cedex 5 13385, France.

Received: 11 February 2013 Accepted: 27 March 2013

Published: 4 April 2013

\section{References}

1. lyer LM, Aravind L, Koonin EV: Common origin of four diverse families of large eukaryotic DNA viruses. J Virol 2001, 75(23):11720-11734.

2. Iyer LM, Balaji S, Koonin EV, Aravind L: Evolutionary genomics of nucleocytoplasmic large DNA viruses. Virus Res 2006, 117(1):156-184.

3. Koonin EV, Yutin N: Origin and evolution of eukaryotic large nucleocytoplasmic DNA viruses. Intervirology 2010, 53(5):284-292.

4. Netherton $\mathrm{CL}$, Wileman $\mathrm{T}$ : Virus factories, double membrane vesicles and viroplasm generated in animal cells. Curr Opin Virol 2011, 1(5):381-387.

5. de Castro IF, Volonte L, Risco C: Virus factories: biogenesis and structural design. Cell Microbiol 2013, 15(1):24-34.

6. Van Etten JL, Dunigan DD: Chloroviruses: not your everyday plant virus. Trends Plant Sci 2012, 17(1):1-8

7. Van Etten JL: Unusual life style of giant chlorella viruses. Annu Rev Genet 2003, 37:153-195.

8. Yutin N, Wolf Yl, Raoult D, Koonin EV: Eukaryotic large nucleo-cytoplasmic DNA viruses: clusters of orthologous genes and reconstruction of viral genome evolution. Virol J 2009, 6:223.

9. Colson P, de Lamballerie X, Fournous G, Raoult D: Reclassification of giant viruses composing a fourth domain of life in the new order Megavirales. Intervirology 2012, 55(5):321-332.

10. Moss B: Poxviridae: the viruses and their replication. In Fields Virology. 2nd edition. Edited by Knipe DM, Howley PM. Philadelphia: Lippincott Williams \& Wilkins; 2007:2905-2946.

11. Claverie JM, Abergel C: Mimivirus: the emerging paradox of quasi-autonomous viruses. Trends Genet 2010, 26(10):431-437.

12. Claverie JM, Ogata H, Audic S, Abergel C, Suhre K, Fournier PE: Mimivirus and the emerging concept of "giant" virus. Virus Res 2006, 117(1):133-144.

13. Raoult $D$, Forterre P: Redefining viruses: lessons from Mimivirus. Nat Rev Microbiol 2008, 6:315-319.

14. Raoult D, Audic S, Robert C, Abergel C, Renesto P, Ogata H, La Scola B, Suzan M, Claverie JM: The 1.2-megabase genome sequence of Mimivirus. Science 2004, 306(5700):1344-1350.

15. Suzan-Monti M, La Scola B, Raoult D: Genomic and evolutionary aspects of Mimivirus. Virus Res 2005, 117(1):145-155.

16. La Scola B, Desnues C, Pagnier I, Robert C, Barrassi L, Fournous G, Merchat M, Suzan-Monti M, Forterre P, Koonin E, et al: The virophage as a unique parasite of the giant mimivirus. Nature 2008, 455(7209):100-104.

17. Claverie JM, Abergel C, Ogata H: Mimivirus. Curr Top Microbiol Immunol 2009, 328:89-121.

18. Colson P, Yutin N, Shabalina SA, Robert C, Fournous G, La Scola B, Raoult D, Koonin EV: Viruses with more than 1,000 genes: Mamavirus, a new Acanthamoeba polyphaga mimivirus strain, and reannotation of Mimivirus genes. Genome Biol Evol 2011, 3:737-742. 
19. Yoosuf N, Yutin N, Colson P, Shabalina SA, Pagnier I, Robert C, Azza S, Klose $\mathrm{T}$, Wong J, Rossmann MG, et al: Related giant viruses in distant locations and different habitats: Acanthamoeba polyphaga moumouvirus represents a third lineage of the Mimiviridae that is close to the megavirus lineage. Genome Biol Evol 2012, 4(12):1324-1330.

20. La Scola B, Campocasso A, N'Dong R, Fournous G, Barrassi L, Flaudrops C, Raoult D: Tentative characterization of new environmental giant viruses by MALDI-TOF mass spectrometry. Intervirology 2010, 53(5):344-353.

21. Arslan D, Legendre M, Seltzer V, Abergel C, Claverie JM: Distant Mimivirus relative with a larger genome highlights the fundamental features of Megaviridae. Proc Natl Acad Sci USA 2011, 108(42):17486-17491.

22. Fischer MG, Allen MJ, Wilson $\mathrm{WH}$, Suttle CA: Giant virus with a remarkable complement of genes infects marine zooplankton. Proc Natl Acad Sci USA 2010, 107(45):19508-19513.

23. Van Etten JL: Another really, really big virus. Viruses 2011, 3(1):32-46.

24. Colson P, Gimenez G, Boyer M, Fournous G, Raoult D: The giant Cafeteria roenbergensis virus that infects a widespread marine phagocytic protist is a new member of the fourth domain of Life. PLoS One 2011, 6(4):e18935.

25. Monier A, Claverie JM, Ogata H: Taxonomic distribution of large DNA viruses in the sea. Genome Biol 2008, 9(7):R106.

26. Monier A, Larsen JB, Sandaa RA, Bratbak G, Claverie JM, Ogata H: Marine mimivirus relatives are probably large algal viruses. Virol J 2008, 5:12

27. Williams TA, Embley TM, Heinz E: Informational gene phylogenies do not support a fourth domain of life for nucleocytoplasmic large DNA viruses. PLoS One 2011, 6(6):e21080

28. Legendre M, Arslan D, Abergel C, Claverie JM: Genomics of Megavirus and the elusive fourth domain of Life. Commun Integr Biol 2012, 5(1):102-106.

29. Nasir A, Kim KM, Caetano-Anolles G: Giant viruses coexisted with the cellular ancestors and represent a distinct supergroup along with superkingdoms Archaea. Bacteria and Eukarya. BMC Evol Biol 2012, 12(1):156.

30. Desnues C, La Scola B, Yutin N, Fournous G, Robert C, Azza S, Jardot P, Monteil S, Campocasso A, Koonin EV, et al: Provirophages and transpovirons as the diverse mobilome of giant viruses. Proc Natl Acad Sci USA 2012, 109(44):18078-18083.

31. Desnues $C$, Boyer M, Raoult D: Sputnik, a virophage infecting the viral domain of life. Adv Virus Res 2012, 82:63-89.

32. Fischer $M G$, Suttle $C A$ : A virophage at the origin of large DNA transposons. Science 2011, 332(6026):231-234.

33. Yau S, Lauro FM, DeMaere MZ, Brown MV, Thomas T, Raftery MJ, AndrewsPfannkoch C, Lewis M, Hoffman JM, Gibson JA, et al: Virophage control of antarctic algal host-virus dynamics. Proc Natl Acad Sci USA 2011, 108(15):6163-6168.

34. Baudoux AC, Brussaard CP: Characterization of different viruses infecting the marine harmful algal bloom species Phaeocystis globosa. Virology 2005, 341(1):80-90

35. Brussard CPG, Bratbak G, Baudoux AC, Ruardij P: Phaeocystis and its interaction with viruses. Biogeochemistry 2007, 83:201-215.

36. Tatusov RL, Koonin EV, Lipman DJ: A genomic perspective on protein families. Science 1997, 278(5338):631-637.

37. Kristensen DM, Wolf YI, Mushegian AR, Koonin EV: Computational methods for Gene Orthology inference. Brief Bioinform 2011, 12(5):379-391.

38. Kristensen DM, Kannan L, Coleman MK, Wolf Yl, Sorokin A, Koonin EV, Mushegian A: A low-polynomial algorithm for assembling clusters of orthologous groups from intergenomic symmetric best matches. Bioinformatics 2010, 26(12):1481-1487.

39. Yutin N, Koonin EV: Proteorhodopsin genes in giant viruses. Biol Direct 2012, 7:34

40. Wolf Yl, Rogozin IB, Grishin NV, Tatusov RL, Koonin EV: Genome trees constructed using five different approaches suggest new major bacterial clades. BMC Evol Biol 2001, 1:8.

41. Yutin N, Koonin EV: Hidden evolutionary complexity of NucleoCytoplasmic Large DNA viruses of eukaryotes. Virol J 2012, 9(1):161.

42. Csuros M: Count: evolutionary analysis of phylogenetic profiles with parsimony and likelihood. Bioinformatics 2010, 26(15):1910-1912.

43. Colson P, Raoult D: Gene repertoire of amoeba-associated giant viruses. Intervirology 2010, 53(5):330-343.

44. Greub G, Raoult D: Microorganisms resistant to free-living amoebae. Clin Microbiol Rev 2004, 17(2):413-433.

45. Raoult D, Boyer M: Amoebae as genitors and reservoirs of giant viruses. Intervirology 2010, 53(5):321-329.
46. Boyer M, Yutin N, Pagnier I, Barrassi L, Fournous G, Espinosa L, Robert C, Azza S, Sun S, Rossmann MG, et al: Giant Marseillevirus highlights the role of amoebae as a melting pot in emergence of chimeric microorganisms. Proc Natl Acad Sci USA 2009, 106(51):21848-21853.

47. Zhou J, Zhang W, Yan S, Xiao J, Zhang Y, Li B, Pan Y, Wang Y: Diversity of virophages in metagenomic datasets. J Virol 2013, 87(8):4225-4236.

48. Altschul SF, Madden TL, Schaffer AA, Zhang J, Zhang Z, Miller W, Lipman DJ: Gapped BLAST and PSI-BLAST: a new generation of protein database search programs. Nucleic Acids Res 1997, 25(17):3389-3402.

49. Edgar RC: MUSCLE: multiple sequence alignment with high accuracy and high throughput. Nucleic Acids Res 2004, 32(5):1792-1797.

50. Marchler-Bauer A, Bryant SH: CD-Search: protein domain annotations on the fly. Nucleic Acids Res 2004, 32(Web Server issue):W327-W331.

51. Felsenstein J: Inferring phylogenies from protein sequences by parsimony, distance, and likelihood methods. Methods Enzymol 1996, 266:418-427.

52. Yutin N, Makarova KS, Mekhedov SL, Wolf YI, Koonin EV: The deep archaeal roots of eukaryotes. Mol Biol Evol 2008, 25(8):1619-1630.

53. Price MN, Dehal PS, Arkin AP: FastTree 2-approximately maximum-likelihood trees for large alignments. PLoS One 2010, 5(3):e9490.

54. Darriba D, Taboada GL, Doallo R, Posada D: ProtTest 3: fast selection of best-fit models of protein evolution. Bioinformatics 2011, 27(8):1164-1165.

55. Jobb G, von Haeseler A, Strimmer K: TREEFINDER: a powerful graphical analysis environment for molecular phylogenetics. BMC Evol Biol $2004,4: 18$

56. Shimodaira $\mathrm{H}$ : An approximately unbiased test of phylogenetic tree selection. Syst Biol 2002, 51(3):492-508.

doi:10.1186/1743-422X-10-106

Cite this article as: Yutin et al:: Mimiviridae: clusters of orthologous genes, reconstruction of gene repertoire evolution and proposed expansion of the giant virus family. Virology Journal 2013 10:106

\section{Submit your next manuscript to BioMed Central and take full advantage of:}

- Convenient online submission

- Thorough peer review

- No space constraints or color figure charges

- Immediate publication on acceptance

- Inclusion in PubMed, CAS, Scopus and Google Scholar

- Research which is freely available for redistribution

Submit your manuscript at www.biomedcentral.com/submit
C Biomed Central 